\title{
NEW CONCEPTS IN PEDIATRIC RHINITIS
}

\author{
Nikos Papadopoulos ${ }^{1}$, Xenophon Aggelides ${ }^{2}$, Sofia Stamataki ${ }^{1}$, Emmanuel Prokopakis ${ }^{3}$, \\ Michael Katotomichelakis ${ }^{4}$, and Paraskevi Xepapadaki ${ }^{1}$ \\ ${ }^{1}$ National and Kapodistrian University of Athens School of Medicine \\ ${ }^{2}$ General University Hospital Attikon \\ ${ }^{3}$ University of Crete School of Medicine \\ ${ }^{4}$ Democritus University of Thrace
}

September 24, 2020

\begin{abstract}
Rhinitis and especially allergic rhinitis (AR) remain the most frequent hypersensitivity condition, affecting up to a quarter of the population and impacting upon the quality of life of individual patients and the health economy. Data, especially in respect to underlying pathophysiological mechanisms mainly derive from adult studies and are subsequently extrapolated in the pediatric population. Therapeutic algorithms for children with rhinitis in children are long based on the same principles as in adults. We explore and describe novel aspects of rhinitis, ranging from mechanisms to disease classification, phenotypes, diagnostic and monitoring tools, and the use of treatments, with focus on the traits of pediatric age groups.
\end{abstract}

\section{Key message}

In this review, we describe novel aspects of pediatric rhinitis, from mechanisms to phenotypes, diagnostic and monitoring tools, and the use of treatments, focusing on the traits of pediatric age groups.

Type 2 innate lymphoid cells and defective Treg cells may play a critical role in the pathogenesis of AR. Recent studies have identified the neural pathways and mediators implicated in nasal hyperreactivity in children, after exposure to environmental factors (pollutants, viruses, allergens). Local allergic rhinitis is present and probably underdiagnosed in pediatric populations. Polysensitization to inhalant allergens is associated with multimorbidity of allergy-associated diseases.

Personalized management of allergic rhinitis relies on the proper assessment of disease control, a combination of multiple measurable parameters with patient-reported outcomes, especially when validated for children, being quite valuable. E-Health applications are revolutionizing healthcare services, but data on their impact on pediatric allergic diseases are scarce.

Second-generation antihistamines for mild and intranasal corticosteroids for moderate-to-severe cases are the mainstay of allergic rhinitis treatment in children. Data from real-world studies have confirmed that allergen immunotherapy can prevent the occurrence of asthma in both adults and children with AR. The need for global policies to mitigate pollution and climate change is more relevant than ever.

\section{Introduction}

Allergic rhinitis (AR) remains the most frequent hypersensitivity condition, affecting up to a quarter of the population and impacting upon the quality of life of individual patients and the health economy as a whole [1]. The complexity and variability of rhinitis is particularly apparent in childhood, during which anatomical, physiological, immunological and microbiological maturation, generates clinical patterns that are difficult to generalize, but also to manage [2]. Nevertheless, in the last few years, new concepts emerging regarding the 
overall understanding and management of rhinitis are having an influence on the way we see pediatric allergic rhinitis today[3]. In this review, we explore and describe novel aspects of rhinitis, ranging from mechanisms to disease classification, phenotypes, diagnostic and monitoring tools and use of treatments, with particular focus on the traits of pediatric age groups.

\section{Mechanisms}

The immunological processes underlying IgE-mediated AR are not dissimilar in children and adults, involving activation of the adaptive immunity and class switching towards production of $\operatorname{IgE}$ antibodies. Nevertheless, the vast amount of evidence derives from adult studies with extrapolation on younger ages, while data from mechanistic studies in children are limited. Of importance, despite innate immunity being present from infancy, quantitative differences and qualitative maturation, potentially affecting downstream responses have been observed from early life though preschool and teenage years [4]. AR, which constitutes the most studied phenotype of Pediatric rhinitis, is predominantly defined by type 2 inflammation, mediated by type 2 innate lymphoid cells (ILC2) and Th2 lymphocyte-driven responses locally and systematically[5]. In addition, infiltration of multiple inflammatory cells in the nasal mucosa including $\mathrm{T}$ cells, eosinophils, neutrophils, and mast cells has been confirmed [6]. Activated/damaged by aeroallergens nasal epithelial cells secrete matrix metalloproteinase, IL-25, IL-31, IL-33 and thymic stromal lymphopoietin (TSLP), which augment Th2 inflammation either via ILC2s or through antigen presenting cells located underneath the nasal epithelium [7]. ILC2s express receptors such as CRTh2, CD127 (the interleukin 7 receptor) and ST-2, the receptor for IL-33 and release particularly IL-9, IL-5 and IL-13, further activating type 2 inflammation. ILC2s also express high levels of GATA3, contributing to CD4+ Th2 activation and subsequent release of IL-4, IL-5, IL-9 and IL-13, which maintain the Th2-cell lineage and furthermore interact with B lymphocytes to induce class-switching and synthesis of allergen-specific IgE (IgE sensitization) [8]. On re-exposure crosslinking of sIgE on effector cells induce the early, within 2-4 hours and subsequently the late phase response characterized predominant by a sustained nasal congestion [9]. In an ex-vivo model, the presence of ILC2s, myeloid and plasmacytoid dendritic cells ( $\mathrm{mDCs}$ and $\mathrm{pDCs}$ respectively) was documented in the nasal mucosa of AR patients. Of importance, mDCs promoted ILC2 function and proliferation by the IL-33/ST2 pathway, while activation of pDCs suppressed ILC2 function through IL-6 in AR patients, suggesting a differential role for distinct dendritic cell subtypes [10].

T regulatory cells (Tregs) are key mediators in immune regulation thus prevent airway allergic inflammation. Inflammation in AR appears to be a functional defect of Tregs which results in decreased production of IL10 and transforming growth factor (TGF) $\beta$ [11]. Certain microRNAs (miRNAs) mediate Treg function [12]. In children with AR, miR-155 promoted Treg differentiation in CD4+ T cells, while other miRNA mediate response to respiratory infections, providing new potential treatment targets [13]. Moreover, other less studied $\mathrm{T}$ cell populations, such as the gammadelta $(\gamma \delta) \mathrm{T}$ cells, that facilitate eosinophil migration, were significantly more abundant in the nasal mucosa of AR patients compared to controls, while their number and distribution were positively associated with eosinophils, macrophages and mast cells locally, suggesting an essential role in mucosal inflammation [14]. Earlier studies showed a dysregulated pattern of Th1 transcription factors such as STAT6+ and GATA3+ potentially contributing to the enhance Th2 responses in AR patients compared to controls [15]. More recently the Notch signaling pathway which downregulates FOXp3 expression and differentiation of T-reg was significantly elevated in AR patients [16].

Studies on the role of B-cells in the pathogenesis of AR, indicate a significant skewing of circulating B-cell population towards memory B cells with markedly increased expression of CD23, which facilitate antigen presentation and IgE synthesis in AR patients [17]; nevertheless, other studies were not confirmatory of differences in B-regulatory cell populations or Il-10 production [18].

Recently, AR patients were shown to exhibit increased number of specific sets of activated neutrophils_CD16(high)-CD62L(dim)_either locally or systematically, resulting in $\mathrm{T}$ cell priming and enhancing eosinophil migration [19]. Recently, activated neutrophils were shown to serve as functional antigen presenting cells (APC) in late phase reactions and activate allergen-specific effector CD4+ T-cells to synthesize cytokines, thus they potentially contribute to magnified nasal allergic inflammation [20]. 
There is evidence that autophagy, a catabolic intracellular process playing a substantial role in maintaining tissue and cellular homeostasis, is implicated in immune response regulation and asthma pathogenesis [21]. More recently, highly expressed autophagic markers were observed in the upper airways of AR patients, strongly associated with indices of nasal remodeling [22].

\section{Phenotypes and endotypes}

The concepts of phenotype and endotype have been increasingly used to characterize variants under a wider disease umbrella, referring to either the apparent differentiating features (phenotype) or the underlying mechanisms (endotype). While disease variability is clearly the norm rather than the exception in regards to most allergic diseases, the realization that treatment response may vary radially between groups, has given impetus to this approach [23]. Unfortunately, lack of pathognomonic biomarkers, considerable overlap and unstable temporal dynamics have not allowed, for the time being, the construction of conclusive phenotype and/or endotype-based guidelines [24]. Nevertheless, clear distinctions and differentiating factors can be drawn, such as the hormonal or gustatory rhinitis - all part of the non-allergic rhinitis spectrum. A distinct pediatric example is rhinitis in infants, which presents additional challenges in management choices, requiring further evidence [25].

\section{Non allergic rhinitis (NAR)}

Non allergic rhinitis in children is poorly defined [26]. Previously AR was clearly discriminated by NAR by means of increased local total/local IgE specific levels and nasal eosinophils and these differences were more pronounced in children than adults with AR, suggesting that AR in children is a more distinct feature [27]. However, inflammatory mediators related to different inflammatory endotypes detected from nasal cytology cannot discriminate AR from NAR adult patients [28].

Local allergic rhinitis (LAR) in children is an emerging disease, highly underdiagnosed [29]. Increased levels of local IgE in the nasal lavage fluid has been proposed as a useful biomarker in children with suspected LAR [30]. Local production of "T2" inflammatory mediators has been demonstrated following nasal allergen challenges in a pediatric setting [31]. Nevertheless, AR and LAR share several clinical and immune features, such as positive response to a nasal allergen provocation test, increased risk of asthma occurrence and increased nasal IgE production [32]. Moreover, the FcepsilonR1beta genetic polymorphism has been linked both with LAR and AR prevalence [33].

Nasal hyperreactivity (NHR) constitutes a cardinal clinical feature both in AR and NAR [34] and has been previously documented in children with allergic rhinitis, although respective techniques are difficult to apply in clinical use and young ages $[35,36]$. The most well described pathogenetic mechanism involves a "dysregulated" neural innervation network. In NAR and NHR, the role of the efferent component via the activated transient receptor potential (TRP) ion channels (receptor 1_TRPA1 and subfamily V-TRPV1), induces the release of neuropeptides, such as CGRP and substance P (SP), neurokinin A and B from sensory nerve endings, which in turn induce sneezing, mucus production and congestion [37]. In line, histological data are indicative of increased expression of the aforementioned neuropeptides of the nasal mucosa in patients with NHR and NAR [38]. Moreover, the impact of environmental and microbial factors (i.e.; staphylococcus Aureus enterotoxins) has been postulated for triggering local nerve endings, more so in the presence of a disrupted epithelium barrier [39]. It remains to be elucidated whether barrier dysfunction is a primary (genetic) defect or secondary to infectious agents or allergens [40, 41].

\section{Multimorbidity}

The term multimorbidity is used to indicate the clustering and co-occurrence of different diseases in an individual, where, conceptually, a "primary" disease in not obvious [42]. Epidemiological data suggest that rhinitis/rhinoconjunctivitis, asthma and dermatitis form a multimorbidity cluster, attributed to shared genetic polymorphisms and pathogenetic mechanisms [43]. The MeDALL (Mechanisms of the Development of ALLergy) project, including more than 12.000 children from 12 ongoing longitudinal cohort studies, clearly showed that co-existence of asthma, rhinitis and eczema in the same child is more prevalent than 
expected by chance [44]. IgE (poly) sensitization was acknowledged as a fundamental component of a specific phenotypical presentation, although not the sole one, significantly associated with disease severity and multimorbidity risk [45]. Moreover, prospective studies indicate that either sensitization per se, or early polysensitization and co-sensitization to both perennial and seasonal allergens, or to furry animals and molds, in case of sole perennial sensitization, are associated with enhanced likelihood of AR patients to present other allergy-associated diseases [46, 47]; however, it is well accepted that a significant proportion of children with IgE sensitization do not develop any allergy-associated disease [48]. In line, molecular studies identified eosinophilic-mediated pathways, including IL-4 and GATA3-related signals, with their respective induced proteins as prominent mediators in allergic multimorbidity [49]; nevertheless, more recently other cell types and pathways have also been implicated in the AR-multimorbidity such as TLR and IL-1 mediated responses [50]. In respect to the implicated genes, several independent risk variants are identified with allergic diseases specific effects have been identified, potentially by dysregulating the expression of lymphocyte-mediated immunity [51]. A recent meta-analysis of worldwide asthma-genome associations studies, verified two loci (8q21.13 and 16p13.13) implicated in the comorbidity of asthma and AR [52].

In respect to allergy-associated disease progression, the MAS study showed that parental history of allergy and broad polymolecular IgE sensitization is a risk factor for presenting allergic multimorbidity later in life [53]. From another perspective, AR combined with ocular and skin allergic diseases is strongly associated with asthma occurrence in adulthood, although this association wanes with age [54]. Sex and puberty-related mechanisms are thought to be related in the "sex shift" prevalence, from male to female predominance after puberty, of allergic multimorbidity, as shown from individual participant data (IPD) meta-analyses in the MeDALL and other cohorts [55]. Of importance, patients with allergic multimorbidity present increased disease burden, drug and healthcare use and costs in all age groups [56].

\section{Control}

In accordance to the WHO proposal for chronic allergic diseases and in parallel to the GINA guidelines for asthma, the concept of control was introduced in AR, as a more feasible measure of both severity and response to treatment $[57,58]$. Most recent AR management algorithms propose a stepwise approach based on the level of control achieved by a therapeutic intervention $[23,59]$. A series of criteria to evaluate control were proposed in the PRACTAL report, including assessing symptom severity, quality of life (QoL) by means of disease burden at school, leisure and sleep, and upper respiratory function by objective measurements such as nasal flow and/or patency. Information regarding rescue medication use and (uncontrolled) comorbidities such as sinusitis and/or asthma are also considered relevant [23].

Patient reported outcome measures (PROMs) are currently acknowledged as valuable tools for assessing disease morbidity in a standard clinical setting. In respect to AR patients the Allergic Rhinitis Control Test (ARCT) [60] and the Pediatric Control of Allergic Rhinitis and Asthma test (CARATkids) [61, 62] have been validated for children and adolescents while the Rhinitis Control Assessment Test (RCAT) is currently validated only for adolescents [63]. The Visual Analogue Scale (VAS) is validated for control assessment only in adults with AR, however it can be used in all ages including preschool children by proxy [64, 65]. Thus, the ARIA algorithm has implemented VAS measurements for daily monitoring in a mobile app for patients over 12 years of age [66]. Nevertheless, it is well known that PROMs can be influenced by poor adherence to treatment, patient's perceptions regarding disease' severity and the individual's psychosocial background [58].

\section{eHealth and mHealth}

eHealth refers to the application of information and communication technologies (ICT) in healthcare services. The WHO acknowledges digital technology potential and encourages health systems to endorse, improve and prioritize its implementation to benefit in terms of accessibility, quality, and affordability of health care [67]. The term "mobile health" (mHealth) refers to the use of mobile, wireless technologies to health practice and research in this respect [68].

Smartphones are most promising in promoting and supporting public health, as they are widely available, 
equipped with sophisticated microprocessors and a range of sensors which can communicate with other mobile devices, and provide access to the internet even in remote locations. As of mid-2020, 3.5 billion smartphones are in use, by approximately $41 \%$ of the world's population [69]. In the USA, $95 \%$ of teens have access to a smartphone and $83 \%$ use it for learning purposes [70].

$\mathrm{AR}$ is often regarded as trivial by both patients and physicians and thus not always managed properly. mHealth applications (apps) aim at implementing personalized medicine strategies to patients and caregivers by monitoring symptoms, visualizing the burden of the disease, thus improving health literacy, and ultimately engaging in self-management and shared decision making [71]. Moreover, eHealth services can override access barriers to healthcare and reduce costs while providing healthcare professionals with real-life data on several aspects of the disease and its impact that otherwise would be difficult to identify.

Adherence to treatment is a common problem in chronic respiratory diseases, such as AR, moreover in adolescents [72]. In the MASK study, where a mobile app was used to assess real-world data from adolescents and adults with AR, non-adherence was observed in $69 \%$ of participants. The effect of e-health services on adherence to nasal steroids was studied in a randomized trial including children 5-18 years old with moderate-severe seasonal AR [73]. Patients that used an internet-based telemonitoring system instead of hard copy diaries, were significantly more adherent to treatment and better educated on their disease, although symptom scores and QoL were not affected. In addition, services such as updates on local pollen counts, online medical access and SMS reminders in case of missed entries can be provided.

mHealth apps are a booming market and caution should be taken on their credibility. Up to day, only a few apps on AR have been validated, while safety and efficacy data are scarce [74]. A recent study showed that the majority of mobile apps providing grass pollen forecasts are inaccurate and potentially harmful [75]. mHealth apps are now acknowledged as medical devices by both the EU and FDA [76], although FDA reserves the concern of potentially applying regulations in case of identifying risk on patient's safety [77]. In Europe, the General Data Protection Regulation is also applied to manufacturers for ensuring consumers' data privacy [78]. A stricter framework of regulations should be expected soon.

Currently, only a few mobile apps that monitor symptoms of both AR and asthma are available [74, 79]. Mask-air and Allergy Monitor allow patients to assess the time course of symptoms relative to treatment and the effect of AR on sleep, productivity, QoL, etc. by sharing information digitally [80]. Additionally, notifications prompt answering clinical questionnaires and treatment adherence daily [81]. In case of symptom worsening, patients are alerted to step up treatment or contact their doctor for consultation. These are key features for encouraging self-awareness and self-empowerment, especially in chronic diseases like AR, where patients or their caregivers are responsible for treatment administration [82]. Mask-air, a validated tool for assessing AR control in adolescents and adults, may also be integrated into a Clinical Decision Support System that guides clinicians into pertinent interventions depending on their patient's status [83]. Evolving algorithms are currently exploring the possibility of data input automation, to improve uptake [84].

\section{Treatment/medications}

Therapeutic algorithms for children with rhinitis in children are long based on the same principles as in adults [85]. During the past two decades ARIA has proposed an evolving guideline for the management of patients with AR. More recently, intergraded care pathways (ICPs) have been proposed, based on technology-evident information, providing a self-care plan by a multidisciplinary group of health professionals centered around the patient [59]. In specific, an algorithm based on the visual analogue scale (VAS) for assessing rhinitis symptoms has been revised by the ARIA expert group for (1) selection of pharmacotherapy for patients with $\mathrm{AR}$ and (2) to tailor treatment depending on control [86].

ARIA has been the first guideline in the field of allergy to have used the GRADE system to formally evaluate and appraise evidence. In its latest update, an important step is the addition or real-life evidence, supported through an mHealth application [59]. Real-life approaches are increasingly used to complement randomized trials, in order to expand the scope and evaluate external validity of the findings [87]. 
Treatment of AR includes allergen avoidance whenever possible, use of medications and allergen specific immunotherapy (AIT)

\section{Environmental interventions}

Certain interventions aiming at reduced allergen (pollen, molds, house dust mites, pets) or irritant (pollutants, tobacco smoke) exposure have long been incorporated into the guidelines [57]. There is substantial evidence that sensitization/exposure to molds correlates significantly with exacerbation of AR symptoms, even in children with non-allergic rhinitis [29, 88]. Mold and cockroach allergen reduction leading to subsequent symptom relief, can be achieved by combined interventions [89]. In general, control of indoor allergen exposure in allergic subjects, is effective when multifaceted and demanding approaches are followed [90]. With regards to house dust mite control, although previous meta-analyses showed minimal protective effects of interventions aiming at exposure reduction, recent data deriving from asthmatic patients indicate that certain actions, such as mite-impermeable bed encasings, may confer significant protection form disease morbidity [91]. It is plausible that early, prolonged and personalized interventions, tailored to individual sensitization and exposure status, can be clinically beneficial [92].

Exposure to AR-relevant outdoor pollens and molds can be reduced by limiting exposure and field activities during peak pollen days, however such recommendation is often unrealistic in the pediatric population and counters the goal of reinforcing daily activity in children [93].

Various epidemiological studies have shown that exposure to volatile organic compounds

from building materials and consumer products exacerbate rhinitis symptoms and although data on the effects on rhinitis of air pollution exposure per se are inconsistent [94, 95], their capacity to increase the allergenicity and immunogenicity of aeroallergens has been shown [96]. Heightened $\mathrm{CO} 2$ levels and global climate change have been strongly associated with increased prevalence of rhinitis symptoms, by increase in pollen levels both in volume and in time; policy adjustments have recently been addressed in order to decrease pollution and mitigate their effects on allergy-associated diseases [97]

\section{Pharmacotherapy}

Medications used for the treatment of AR in children include non-sedating H1-antihistamines, leukotriene receptor antagonists, chromones, intranasal corticosteroids (INCSs) and combination of INCs and azelastine.

\section{Antihistamines}

Antihistamines are available as oral, intranasal, and ocular preparations. Oral first generation, sedating antihistamines are no longer recommended for the treatment of pediatric rhinitis due to their impaired safety profile often associated with cardiac arrhythmias, drowsiness, confusion, vision, and mental disorders etc. Of importance, a recent report using data from the World Health Organization (WHO) database, showed that the use of certain antihistamines has been strongly associated with severe and unexpected reactions, more so in preschoolers, where off-label use is frequent [98]. Second generation antihistamines present a significantly improved safety profile due to their high H1-receptor selectivity, low brain permeability and longer duration of action [99]. Recently, cardiac rhythm safety was documented for certain $2^{\text {nd }}$ generation antihistamines, such as bilastine, cetirizine, levocetirizine, ebastine, fexofenadine, loratadine, desloratadine, mizolastine and rupatadine, even in the case of quadrupling the recommended standard dose [100]. Antihistamines, act predominantly on neutrally mediated symptoms such as sneeze, rhinorrhea, and nasal itch. They should be used continuously, rather than no-demand in persistent rhinitis patients [93]. Topical/nasal antihistamines have rapid onset of action and minimum side effects [101]. In addition, azelastine has been shown to have mast cell-stabilizing, anti-leukotriene, anti-inflammatory and immunomodulatory effects [102].

\section{Intranasal corticosteroids (INCs)}

INSc are the mainstay, particularly for moderate-to-severe allergic rhinitis, due to their anti-inflammatory properties and significant attenuation of late phase allergic reaction, thus acting on nasal congestion [59, 103]. In respect to non-allergic rhinitis, a recent Cochrane review showed that INCs use has only short term 
beneficial effects on symptom severity, potentially depending on individual phenotypic characteristics [104]. INCs have a rapid onset of action, while the newer preparations such as mometasone furoate, fluticasone furoate (FP) and fluticasone propionate present negligible systemic absorption, thus they are mostly suitable for children [105]. On-demand use can be an efficacious alternative in children with seasonal AR symptoms, especially in families with safety concerns on daily CS use, in order to increase adherence [106]. Recently, an ARIA-EAACI statement suggested that, based on current evidence, patients with COVID-19 infection should not discontinue their INCS prescribed for AR symptoms [107].

The recently formulated combination of azelastine and fluticasone propionate (FP) has shown short and long term efficacy, with superiority over FP both in speed of action and symptom relief, in children with AR $[108,109]$. Safety and tolerability have been documented in children over 4-years of age, with minimal local adverse reactions such as dysgeusia [110]. The combination has been granted approval for use in children over 6 years of age by the U.S. Food and Drug Administration [111].

Antagonists of leukotriene receptors (LTRA) block cysteinyl leukotrienes receptors, which are important mediators of the inflammatory response in both AR and asthma [112]. Several studies have shown inferiority of LTRA compared to INCS and SGHA as therapeutic choices in AR, while as of 03/04/2020 FDA required a boxed warning, based on limited data reporting serious mental health side effects, thus restricting montelukast use for AR [113].

\section{Biologicals}

Uncontrolled and severe forms of AR with substantial impact on quality of life, have been considered as a potential target for biologicals [114]. Recent reports and meta-analysis showed that anti-IgE (omalizumab) significantly reduces rhinitis morbidity, severity and rescue medication use and improve quality of life in uncontrolled adolescents $[115,116]$. There are also data indicating that pre-treatment with anti-IgE in children undergoing seasonal AIT, significantly decrease severe adverse reactions and improves daily rhinitis symptoms [117]; nevertheless, Omalizumab is not indicated in AR due to cost limitation.

Dupilumab is an anti-IL-4 monoclonal antibody currently approved for uncontrolled atopic dermatitis and asthma [118]. In a Phase IIb clinical trial dupilumab was efficient in ameliorating rhinitis symptoms such as postnasal discharge, nasal blockage, runny nose and sneezing, in patients with uncontrolled asthma and perennial AR [119]. The results from a clinical study evaluating the efficacy of dupilumab as an adjuvant therapy in patients undergoing AIT are awaited[120].

\section{Alternative therapies}

Nasal saline irrigation has long been perceived as an adjunct treatment for rhinitis, although efficacy was only recently documented in a meta-analysis with no reported adverse reactions [121]. Recently, a real life study in children showed significant improvement of AR symptoms and reduction of medications, as these were assessed by VAS and CARAT, when nasal irrigation was added to standard treatment [122].

Use of nasal filters in AR adults with grass allergy during a natural in season exposure, provided significant efficacy, by means of improved nasal symptom score, while safety and usability were also documented [123]. It is however uncertain whether such intervention may be tolerable in children. In respect to other 'alternative' treatments, acupuncture has moderate to high evidence of efficacy for the treatment of subjects with rhinitis, while butterbar has shown showed moderate beneficial effects on AR symptoms [124]. Capsaicin is efficient for the treatment of non-AR in adults; however pediatric studies are lacking [125].

\section{Immunotherapy}

Allergen immunotherapy (AIT), either in the subcutaneous (SCIT) or sublingual (SLIT) form, has long been shown to result in improved AR control in all age groups and aspires to be the only etiologic treatment for respiratory allergies (allergic rhinitis (AR) and asthma) through induction of immunologic tolerance [126] [127]. Pooled analyses support the complementary use of AIT on pharmacotherapy in AR patients moreover due to achievement of long-lasting therapeutic effect [128]. Recently, data from a real-world study confirmed 
the medication sparing effect and long-term symptom relief following AIT, in a cohort of grass AR patients, including children. In agreement, subcutaneous allergoid immunotherapy in house dust mite AR children resulted in significant reduction in AR and asthma medication, even after long-term cessation of AIT [129].

Early-onset asthma is largely associated with IgE sensitization and AR [130], thus AIT has long been suggested as a disease modifying strategy potentially preventing allergen sensitization and asthma occurrence in children with AR [131]. Although early clinical studies showed a preventive effect of AIT on the occurrence of new sensitizations in children with rhinitis [132], this is still inconclusive [133]. A recent meta-analysis identified a short term preventive effect of AIT on novel sensitization development; nevertheless, the included studies were highly heterogenous [134]. Nevertheless, the preventive effects of AIT on asthma development and progression has been confirmed in real-world studies, assessing the effect of SLIT immunotherapy in grass pollen subjects with AR [135]. Moreover, a long-term randomized controlled trial, the Grazax Asthma Prevention (GAP) study, [136], showed significantly lower and long-lasting incidence of asthma following 3year AIT in grass AR subjects, while significant lower levels of total and allergen specific IgE and reductions in skin prick test response to grass were noted [137].

\section{Conclusion}

While the amount of knowledge on rhinitis and allergic rhinitis in children is continuously increasing, novel approaches mostly deriving from adult populations, have the potential of improving outcomes in children. Considering that blinded randomized trials are more difficult to perform in pediatrics, real-life approaches may be used to confirm the usefulness of these proposals[138]. It is probable that, as in the case of pediatric asthma [139], prevention studies and interventions on the natural history of rhinitis and/or respiratory allergy as a whole, are the highest priority for research and health policy.

\section{REFERENCES}

1. Samoliński, B., et al., Prevention and control of childhood asthma and allergy in the EU from the public health point of view: Polish Presidency of the European Union. Allergy, 2012.67 (6): p. 726-31.

2. Georgountzou, A., et al., Age-dependent changes in circulating leukocytes from birth to adulthood in healthy and atopic subjects.Allergy, 2019. 74 (S106): p. 139-139.

3. Fokkens, W.J., et al., European Position Paper on Rhinosinusitis and Nasal Polyps 2020. Rhinology, 2020.58 (Suppl S29): p. 1-464.

4. Georgountzou, A. and N.G. Papadopoulos, Postnatal Innate Immune Development: From Birth to Adulthood. Front Immunol, 2017. 8 : p. 957.

5. Zhong, H., et al., Increased innate type 2 immune response in house dust mite-allergic patients with allergic rhinitis. Clin Immunol, 2017. 183 : p. 293-299.

6. Hammad, H. and B.N. Lambrecht, Barrier Epithelial Cells and the Control of Type 2 Immunity. Immunity, 2015. 43 (1): p. 29-40.

7. Divekar, R. and H. Kita, Recent advances in epithelium-derived cytokines (IL-33, IL-25, and thymic stromal lymphopoietin) and allergic inflammation. Curr Opin Allergy Clin Immunol, 2015. 15 (1): p. 98-103.

8. Wise, S.K., et al., International Consensus Statement on Allergy and Rhinology: Allergic Rhinitis. Int Forum Allergy Rhinol, 2018. 8 (2): p. 108-352.

9. Eifan, A.O. and S.R. Durham, Pathogenesis of rhinitis. Clin Exp Allergy, 2016. 46 (9): p. 1139-51.

10. Peng, Y.Q., et al., Effects of myeloid and plasmacytoid dendritic cells on ILC2s in patients with allergic rhinitis. J Allergy Clin Immunol, 2020. 145 (3): p. 855-867 e8.

11. Coomes, S.M., et al., CD4(+) Th2 cells are directly regulated by $I L-10$ during allergic airway inflammation. Mucosal Immunol, 2017.10 (1): p. 150-161. 
12. Taka, S., et al., MicroRNAs in Asthma and Respiratory Infections: Identifying Common Pathways. Allergy Asthma Immunol Res, 2020. 12 (1): p. 4-23.

13. Zeng, Q., et al., MicroRNA-181a and microRNA-155 are involved in the regulation of the differentiation and function of regulatory $T$ cells in allergic rhinitis children. Pediatr Allergy Immunol, 2019.30 (4): p. 434-442.

14. Yang, Q., et al., Infiltration pattern of gammadelta $T$ cells and its association with local inflammatory response in the nasal mucosa of patients with allergic rhinitis. Int Forum Allergy Rhinol, 2019.9 (11): p. 1318-1326.

15. Eifan, A.O., et al., Reduced T-bet in addition to enhanced STAT6 and GATA3 expressing T cells contribute to human allergen-induced late responses. Clin Exp Allergy, 2012. 42 (6): p. 891-900.

16. Jiao, W.E., et al., Notch Signaling Promotes Development of Allergic Rhinitis by Suppressing Foxp3 Expression and Treg Cell Differentiation. Int Arch Allergy Immunol, 2019. 178 (1): p. 33-44.

17. Yao, Y., et al., CD23 expression on switched memory B cells bridges $T$-B cell interaction in allergic rhinitis. Allergy, 2020.

18. Wirz, O.F., et al., Comparison of regulatory B cells in asthma and allergic rhinitis. Allergy, 2019. 74 (4): p. $815-818$.

19. Arebro, J., et al., A possible role for neutrophils in allergic rhinitis revealed after cellular subclassification. Sci Rep, 2017. 7 : p. 43568.

20. Polak, D., et al., A novel role for neutrophils in IgE-mediated allergy: Evidence for antigen presentation in late-phase reactions. J Allergy Clin Immunol, 2019. 143 (3): p. 1143-1152 e4.

21. Kota, A., et al., Autophagy and airway fibrosis: Is there a link? F1000Res, 2017. 6 : p. 409.

22. Li, J. and Y. Li, Autophagy is involved in allergic rhinitis by inducing airway remodeling. Int Forum Allergy Rhinol, 2019.9 (11): p. 1346-1351.

23. Papadopoulos, N.G., et al., Phenotypes and endotypes of rhinitis and their impact on management: a PRACTALL report. Allergy, 2015. 70 (5): p. 474-94.

24. Papadopoulos, N.G. and G.V. Guibas, Rhinitis Subtypes, Endotypes, and Definitions. Immunol Allergy Clin North Am, 2016.36 (2): p. 215-33.

25. Patel, V.A. and M.M. Carr, Congenital nasal obstruction in infants: A retrospective study and literature review. Int J Pediatr Otorhinolaryngol, 2017. 99 : p. 78-84.

26. Poddighe, D., et al., Non-allergic rhinitis in children: Epidemiological aspects, pathological features, diagnostic methodology and clinical management. World J Methodol, 2016. 6 (4): p. 200-213.

27. Lee, K.S., et al., Local Immune Responses in Children and Adults with Allergic and Nonallergic Rhinitis. PLoS One, 2016.11 (6): p. e0156979.

28. Segboer, C.L., et al., Endotyping of non-allergic, allergic and mixed rhinitis patients using a broad panel of biomarkers in nasal secretions. PLoS One, 2018. 13 (7): p. e0200366.

29. Tsilochristou, O., et al., Detection of local allergic rhinitis in children with chronic, difficult-to-treat, non-allergic rhinitis using multiple nasal provocation tests. Pediatr Allergy Immunol, 2019. 30 (3): p. 296-304.

30. Colavita, L., et al., Local Allergic Rhinitis in Pediatric Patients: Is IgE Dosage in Nasal Lavage Fluid a Useful Diagnostic Method in Children? Int J Mol Cell Med, 2017. 6 (3): p. 174-182.

31. Zicari, A.M., et al., Local allergic rhinitis in children: Novel diagnostic features and potential biomarkers. Am J Rhinol Allergy, 2016. 30 (5): p. 329-34. 
32. Campo, P., et al., Local allergic rhinitis: Implications for management. Clin Exp Allergy, 2019. 49 (1): p. 6-16.

33. NE, E.L. and M.W. El-Anwar, Assessment of Nasal Immunoglobulin E Level in Atopic and Non-atopic Rhinitis Patients: A Tool for Diagnosis of Local Allergic Rhinitis. Egypt J Immunol, 2016. 23 (1): p. 45-56.

34. Van Gerven, L., B. Steelant, and P.W. Hellings, Nasal hyperreactivity in rhinitis: A diagnostic and therapeutic challenge.Allergy, 2018. 73 (9): p. 1784-1791.

35. Giannico, R., et al., Non-specific nasal provocation in children with chronic allergic rhinitis. J Investig Allergol Clin Immunol, 1996. 6 (2): p. 110-6.

36. Lluch-Bernal, M., et al., Nasal Hyperreactivity: Nonspecific Nasal Provocation Tests. Review by the Rhinoconjunctivitis Committee of the Spanish Society of Allergy and Clinical Immunology. J Investig Allergol Clin Immunol, 2015. 25 (6): p. 396-407.

37. Baroody, F.M., Nonallergic Rhinitis: Mechanism of Action.Immunol Allergy Clin North Am, 2016. 36 (2): p. 279-87.

38. Van Gerven, L., et al., Capsaicin treatment reduces nasal hyperreactivity and transient receptor potential cation channel subfamily $V$, receptor 1 (TRPV1) overexpression in patients with idiopathic rhinitis. J Allergy Clin Immunol, 2014. 133 (5): p. 1332-9, 1339 e1-3.

39. Steelant, B., et al., Impaired barrier function in patients with house dust mite-induced allergic rhinitis is accompanied by decreased occludin and zonula occludens-1 expression. J Allergy Clin Immunol, 2016. 137 (4): p. 1043-1053 e5.

40. Hamed, A., et al., Nasal IgE production in allergic rhinitis: Impact of rhinovirus infection. Clin Exp Allergy, 2019. 49 (6): p. 847-852.

41. Doulaptsi, M., et al., Prevalence and impact of nasal hyperreactivity in chronic rhinosinusitis. Allergy, 2020.75 (7): p. 1768-1771.

42. Bousquet, J., et al., Are allergic multimorbidities and IgE polysensitization associated with the persistence or re-occurrence of foetal type 2 signalling? The MeDALL hypothesis. Allergy, 2015.70 (9): p. 1062-78.

43. Anto, J.M., et al., Mechanisms of the Development of Allergy (MeDALL): Introducing novel concepts in allergy phenotypes. J Allergy Clin Immunol, 2017. 139 (2): p. 388-399.

44. Pinart, M., et al., Comorbidity of eczema, rhinitis, and asthma in IgE-sensitised and non-IgE-sensitised children in MeDALL: a population-based cohort study. Lancet Respir Med, 2014. 2 (2): p. 131-40.

45. Bousquet, J., et al., MeDALL (Mechanisms of the Development of ALLergy): an integrated approach from phenotypes to systems medicine.Allergy, 2011. 66 (5): p. 596-604.

46. Blondal, V., et al., Study of atopic multimorbidity in subjects with rhinitis using multiplex allergen component analysis.Clin Transl Allergy, 2020. 10 : p. 6.

47. Gabet, S., et al., Early polysensitization is associated with allergic multimorbidity in PARIS birth cohort infants. Pediatr Allergy Immunol, 2016. 27 (8): p. 831-837.

48. Ballardini, N., et al., IgE antibodies in relation to prevalence and multimorbidity of eczema, asthma, and rhinitis from birth to adolescence. Allergy, 2016. 71 (3): p. 342-9.

49. Aguilar, D., et al., Computational analysis of multimorbidity between asthma, eczema and rhinitis. PLoS One, 2017. 12 (6): p. e0179125.

50. Aguilar, D., et al., Understanding allergic multimorbidity within the non-eosinophilic interactome. PLoS One, 2019.14 (11): p. e0224448. 
51. Ferreira, M.A., et al., Shared genetic origin of asthma, hay fever and eczema elucidates allergic disease biology. Nat Genet, 2017.49 (12): p. 1752-1757.

52. Demenais, F., et al., Multiancestry association study identifies new asthma risk loci that colocalize with immune-cell enhancer marks. Nat Genet, 2018. 50 (1): p. 42-53.

53. Lau, S., et al., Allergy and atopy from infancy to adulthood: Messages from the German birth cohort MAS. Ann Allergy Asthma Immunol, 2019. 122 (1): p. 25-32.

54. Toppila-Salmi, S., et al., Risk of adult-onset asthma increases with the number of allergic multimorbidities and decreases with age. Allergy, 2019. 74 (12): p. 2406-2416.

55. Hohmann, C., et al., Sex-specific incidence of asthma, rhinitis and respiratory multimorbidity before and after puberty onset: individual participant meta-analysis of five birth cohorts collaborating in MeDALL. BMJ Open Respir Res, 2019. 6 (1): p. e000460.

56. Jantunen, J., et al., Multimorbidity in Asthma, Allergic Conditions and COPD Increase Disease Severity, Drug Use and Costs: The Finnish Pharmacy Survey. Int Arch Allergy Immunol, 2019.179 (4): p. 273-280.

57. Bousquet, J., et al., Allergic Rhinitis and its Impact on Asthma (ARIA) 2008 update (in collaboration with the World Health Organization, GA(2)LEN and AllerGen). Allergy, 2008. 63 Suppl 86 : p. 8-160.

58. Demoly, P., et al., Assessment of disease control in allergic rhinitis. Clin Transl Allergy, 2013. 3 (1): p. 7 .

59. Bousquet, J., et al., Next-generation Allergic Rhinitis and Its Impact on Asthma (ARIA) guidelines for allergic rhinitis based on Grading of Recommendations Assessment, Development and Evaluation (GRADE) and real-world evidence. J Allergy Clin Immunol, 2020.145 (1): p. 70-80 e3.

60. Demoly, P., et al., Validation of a self-questionnaire for assessing the control of allergic rhinitis. Clin Exp Allergy, 2011.41 (6): p. 860-8.

61. Linhares, D.V., et al., Validation of control of allergic rhinitis and asthma test for children (CARATKids)-a prospective multicenter study. Pediatr Allergy Immunol, 2014. 25 (2): p. 173-9.

62. Emons, J.A., et al., Use of the Control of Allergic Rhinitis and Asthma Test (CARATkids) in children and adolescents: Validation in Dutch. Pediatr Allergy Immunol, 2017. 28 (2): p. 185-190.

63. Meltzer, E.O., et al., Reliability, validity, and responsiveness of the Rhinitis Control Assessment Test in patients with rhinitis. J Allergy Clin Immunol, 2013. 131 (2): p. 379-86.

64. Morais-Almeida, M., et al., Prevalence and classification of rhinitis in preschool children in Portugal: a nationwide study.Allergy, 2013. 68 (10): p. 1278-88.

65. Bousquet, J., et al., ARIA 2016: Care pathways implementing emerging technologies for predictive medicine in rhinitis and asthma across the life cycle. Clin Transl Allergy, 2016. 6 : p. 47.

66. Bousquet, J., et al., MACVIA clinical decision algorithm in adolescents and adults with allergic rhinitis. J Allergy Clin Immunol, 2016. 138 (2): p. 367-374 e2.

67. World Health Organization (WHO). Digital Health . 2018 April 12,2020]; Available from:https://apps.who.int/gb/ebwha/pdf_files/WHA71/A71_R7-en.pdf?ua=1.

68. World Health Organization (WHO). Recommendations on digital interventions for health system strengthening . 2019 [cited 2020 April 20]; Available from:https://apps.who.int/iris/bitstream/handle/10665/311941/9789241550505-eng.pdf?ua=1.

69. Gu, T. Newzoo's Global Mobile Market Report: Insights into the World's 3.2 Billion Smartphone Users, the Devices They Use $\&$ the Mobile Games They Play . 2019; 
Available from:https://newzoo.com/insights/articles/newzoos-global-mobile-market-report-insights-into-theworlds-3-2-billion-smartphone-users-the-devices-they-use-the-mobile-games-they-play/.

70. Schaeffer, K. Most U.S. teens who use cellphones do it to pass time, connect with others, learn new things . 2019 [cited 2020 April 21, 2020]; Available from:https://pewresearch-org-preprod.go-vip.co/facttank/2019/08/23/most-u-s-teens-who-use-cellphones-do-it-to-pass-time-connect-with-others-learn-newthings/.

71. Bousquet, J., et al., Allergic Rhinitis and its Impact on Asthma (ARIA) Phase 4 (2018): Change management in allergic rhinitis and asthma multimorbidity using mobile technology. J Allergy Clin Immunol, 2019. 143 (3): p. 864-879.

72. Gray, W.N., et al., Medication adherence in pediatric asthma: A systematic review of the literature. Pediatr Pulmonol, 2018.53 (5): p. 668-684.

73. Pizzulli, A., et al., The impact of telemonitoring on adherence to nasal corticosteroid treatment in children with seasonal allergic rhinoconjunctivitis. Clin Exp Allergy, 2014. 44 (10): p. 1246-54.

74. Matricardi, P.M., et al., The role of mobile health technologies in allergy care: An EAACI position paper. Allergy, 2020.75 (2): p. 259-272.

75. Bastl, K., U. Berger, and M. Kmenta, Evaluation of Pollen Apps Forecasts: The Need for Quality Control in an eHealth Service. J Med Internet Res, 2017. 19 (5): p. e152.

76. European Council, Regulation 2017/745 of the European Parliament and of the Council of 5 April 2017 on medical devices. Official Journal of the European Union, 2017. 60 : p. 1-175.

77. U.S. Food \& Drug Administration (FDA). Policy for Device Software Functions and Mobile Medical Applications . 2019 [cited 2020 April 21]; Available from:https://www.fda.gov/media/80958/download.

78. European Council, Regulation 2016/679 of the European Parliament and of the Council of 27 April 2016 on the protection of natural persons with regard to the processing of personal data and on the free movement of such data, and repealing Directive 95/46/EC (General Data Protection Regulation). Official Journal of the European Union, 2016. 59 : p. 1-88.

79. Bousquet, J., et al., Mobile Technology in Allergic Rhinitis: Evolution in Management or Revolution in Health and Care? J Allergy Clin Immunol Pract, 2019. 7 (8): p. 2511-2523.

80. Caimmi, D., et al., Validation of the MASK-rhinitis visual analogue scale on smartphone screens to assess allergic rhinitis control. Clin Exp Allergy, 2017. 47 (12): p. 1526-1533.

81. Bousquet, J., et al., MASK 201\%: ARIA digitally-enabled, integrated, person-centred care for rhinitis and asthma multimorbidity using real-world-evidence. Clin Transl Allergy, 2018. 8 : p. 45.

82. Sleurs, K., et al., Mobile health tools for the management of chronic respiratory diseases. Allergy, 2019. 74 (7): p. 1292-1306.

83. Courbis, A.L., et al., Electronic Clinical Decision Support System for allergic rhinitis management: MASK e-CDSS. Clin Exp Allergy, 2018. 48 (12): p. 1640-1653.

84. L., K., et al., Allergymap: A Hybrid mHealth Mobile Crowdsensing System for Allergic Diseases Epidemiology : a multidisciplinary case study, in 2018 IEEE International Conference on Pervasive Computing and Communications Workshops (Percom Workshops) . 2018, IEEE: Athens. p. 597-602.

85. Roberts, G., et al., Paediatric rhinitis: position paper of the European Academy of Allergy and Clinical Immunology. Allergy, 2013.68 (9): p. 1102-16.

86. Klimek, L., et al., Visual analogue scales (VAS): Measuring instruments for the documentation of symptoms and therapy monitoring in cases of allergic rhinitis in everyday health care: Position Paper of the German Society of Allergology (AeDA) and the German Society of Allergy and Clinical Immunology (DGAKI), 
ENT Section, in collaboration with the working group on Clinical Immunology, Allergology and Environmental Medicine of the German Society of Otorhinolaryngology, Head and Neck Surgery (DGHNOKHC). Allergo J Int, 2017. 26 (1): p. 16-24.

87. Roche, N., et al., The importance of real-life research in respiratory medicine: manifesto of the Respiratory Effectiveness Group: Endorsed by the International Primary Care Respiratory Group and the World Allergy Organization. Eur Respir J, 2019. 54 (3).

88. Katotomichelakis, M., et al., Alternaria and Cladosporium calendar of Western Thrace: Relationship with allergic rhinitis symptoms. Laryngoscope, 2016. 126 (2): p. E51-6.

89. Cox, L., Approach to Patients with Allergic Rhinitis: Testing and Treatment. Med Clin North Am, 2020. 104 (1): p. 77-94.

90. Phipatanakul, W., et al., Environmental assessment and exposure reduction of rodents: a practice parameter. Ann Allergy Asthma Immunol, 2012. 109 (6): p. 375-87.

91. Murray, C.S., et al., Preventing Severe Asthma Exacerbations in Children. A Randomized Trial of Mite-Impermeable Bedcovers. Am J Respir Crit Care Med, 2017. 196 (2): p. 150-158.

92. Custovic, A., C.S. Murray, and A. Simpson, Dust-mite inducing asthma: what advice can be given to patients? Expert Rev Respir Med, 2019. 13 (10): p. 929-936.

93. Scadding, G.K., et al., BSACI guideline for the diagnosis and management of allergic and non-allergic rhinitis (Revised Edition 2017; First edition 2007). Clin Exp Allergy, 2017. 47 (7): p. 856-889.

94. Nurmatov, U.B., et al., Volatile organic compounds and risk of asthma and allergy: a systematic review. Eur Respir Rev, 2015.24 (135): p. 92-101.

95. Burte, E., et al., Association between air pollution and rhinitis incidence in two European cohorts. Environ Int, 2018.115 : p. 257-266.

96. Zhao, F., et al., Common ragweed (Ambrosia artemisiifolia L.): allergenicity and molecular characterization of pollen after plant exposure to elevated NO2. Plant Cell Environ, 2016. 39 (1): p. 147-64.

97. Eguiluz-Gracia, I., et al., The need for clean air: The way air pollution and climate change affect allergic rhinitis and asthma.Allergy, 2020.

98. Motola, D., et al., Safety profile of H1-antihistamines in pediatrics: an analysis based on data from VigiBase. Pharmacoepidemiol Drug Saf, 2017. 26 (10): p. 1164-1171.

99. Church, M.K., Allergy, Histamine and Antihistamines. Handb Exp Pharmacol, 2017. 241 : p. 321-331.

100. Cataldi, M., et al., Cardiac safety of second-generation H1 -antihistamines when updosed in chronic spontaneous urticaria. Clin Exp Allergy, 2019. 49 (12): p. 1615-1623.

101. Horak, F. and U.P. Zieglmayer, Azelastine nasal spray for the treatment of allergic and nonallergic rhinitis. Expert Rev Clin Immunol, 2009. 5 (6): p. 659-69.

102. Schumacher, S., et al., Unique immunomodulatory effects of azelastine on dendritic cells in vitro. Naunyn Schmiedebergs Arch Pharmacol, 2014. 387 (11): p. 1091-9.

103. Karatzanis, A., et al., Contemporary Use of Corticosteroids in Rhinology. Curr Allergy Asthma Rep, 2017. 17 (2): p. 11.

104. Segboer, C., et al., Intranasal corticosteroids for non-allergic rhinitis. Cochrane Database Syst Rev, 2019.2019 (11).

105. Bensch, G.W., Safety of intranasal corticosteroids. Ann Allergy Asthma Immunol, 2016. 117 (6): p. 601-605. 
106. Hicks, A. and M. Egan, On-Demand Intranasal Corticosteroids for Treatment of Seasonal Allergic Rhinitis in Children. J Allergy Clin Immunol Pract, 2018. 6 (4): p. 1430-1431.

107. Bousquet, J., et al., Intranasal corticosteroids in allergic rhinitis in COVID-19 infected patients: An ARIA-EAACI statement.Allergy, 2020.

108. Berger, W., et al., Efficacy of MP-AzeFlu in children with seasonal allergic rhinitis: Importance of paediatric symptom assessment. Pediatr Allergy Immunol, 2016. 27 (2): p. 126-33.

109. Debbaneh, P.M., et al., Intranasal Azelastine and Fluticasone as Combination Therapy for Allergic Rhinitis: Systematic Review and Meta-analysis. Otolaryngol Head Neck Surg, 2019. 161 (3): p. 412-418.

110. Berger, W., et al., Safety of a novel intranasal formulation of azelastine hydrochloride and fluticasone propionate in children: A randomized clinical trial. Allergy Asthma Proc, 2018. 39 (2): p. 110-116.

111. http://cisn.co/2AD11JK.,Dymista approved in children 6 to 11 years of age with seasonal allergic rhinitis . Accessed 12 Oct 2018.

112. Schuler Iv, C.F. and J.M. Montejo, Allergic Rhinitis in Children and Adolescents. Pediatr Clin North Am, 2019. 66 (5): p. 981-993.

113. (FDA), U.S.F.D.A. FDA requires Boxed Warning about serious mental health side effects for asthma and allergy drug montelukast (Singulair); advises restricting use for allergic rhinitis . 2020 [cited 2020 $03 \mathrm{Sep}]$; Available from:https://www.fda.gov/drugs/drug-safety-and-availability/fda-requires-boxed-warningabout-serious-mental-health-side-effects-asthma-and-allergy-drug.

114. Licari, A., et al., Current and emerging biologic therapies for allergic rhinitis and chronic rhinosinusitis. Expert Opin Biol Ther, 2020: p. 1-11.

115. Humbert, M., et al., IgE-Mediated Multimorbidities in Allergic Asthma and the Potential for Omalizumab Therapy. J Allergy Clin Immunol Pract, 2019. 7 (5): p. 1418-1429.

116. Yu, C., et al., Clinical Efficacy and Safety of Omalizumab in the Treatment of Allergic Rhinitis: A Systematic Review and Meta-analysis of Randomized Clinical Trials. Am J Rhinol Allergy, 2020.34 (2): p. 196-208.

117. Kopp, M.V., et al., Combination of omalizumab and specific immunotherapy is superior to immunotherapy in patients with seasonal allergic rhinoconjunctivitis and co-morbid seasonal allergic asthma.Clin Exp Allergy, 2009. 39 (2): p. 271-9.

118. Castro, M., et al., Dupilumab Efficacy and Safety in Moderate-to-Severe Uncontrolled Asthma. N Engl J Med, 2018.378 (26): p. 2486-2496.

119. Weinstein, S.F., et al., Efficacy and safety of dupilumab in perennial allergic rhinitis and comorbid asthma. J Allergy Clin Immunol, 2018. 142 (1): p. 171-177 e1.

120. Dupilumab As An Adjunct For Subcutaneous Grass Immunotherapy. ClinicalTrials.gov identifier:NCT03558997.https://ClinicalTrials.gov/show/NCT03558997. Updated May 28, 2020. Accessed September 3, 2020.

121. Head, K., et al., Saline irrigation for allergic rhinitis.Cochrane Database Syst Rev, 2018. 6 : p. CD012597.

122. Mitsias, D.I., et al., Effect of nasal irrigation on allergic rhinitis control in children; complementarity between CARAT and MASK outcomes. Clin Transl Allergy, 2020. 10 : p. 9.

123. Kenney, P., et al., Preventive effect of nasal filters on allergic rhinitis: A randomized, double-blind, placebo-controlled crossover park study. J Allergy Clin Immunol, 2015. 136 (6): p. 1566-1572 e5. 
124. Wu, A.W., et al., Alternative therapies for sinusitis and rhinitis: a systematic review utilizing a modified Delphi method. Int Forum Allergy Rhinol, 2020.

125. Fokkens, W., P. Hellings, and C. Segboer, Capsaicin for Rhinitis. Curr Allergy Asthma Rep, 2016. 16 (8): p. 60 .

126. Halken, S., et al., EAACI guidelines on allergen immunotherapy: Prevention of allergy. Pediatr Allergy Immunol, 2017.28 (8): p. 728-745.

127. Mitsias, D.I., et al., Immunotherapy in allergic diseases - improved understanding and innovation for enhanced effectiveness. Curr Opin Immunol, 2020. 66 : p. 1-8.

128. Durham, S.R., et al., Treatment effect of sublingual immunotherapy tablets and pharmacotherapies for seasonal and perennial allergic rhinitis: Pooled analyses. J Allergy Clin Immunol, 2016.138 (4): p. 1081-1088 e4.

129. Jutel, M., et al., Real-world evidence of subcutaneous allergoid immunotherapy in house dust miteinduced allergic rhinitis and asthma. Allergy, 2020.

130. Papadopoulos, N.G., et al., International consensus on (ICON) pediatric asthma. Allergy, 2012. 67 (8): p. $976-97$.

131. Pitsios, C., et al., Clinical contraindications to allergen immunotherapy: an EAACI position paper. Allergy, 2015. 70 (8): p. 897-909.

132. Pajno, G.B., et al., Prevention of new sensitizations in asthmatic children monosensitized to house dust mite by specific immunotherapy. A six-year follow-up study. Clin Exp Allergy, 2001.31 (9): p. 1392-7.

133. Lim, J.H., et al., Sublingual immunotherapy (SLIT) for house dust mites does not prevent new allergen sensitization and bronchial hyper-responsiveness in allergic rhinitis children. PLoS One, 2017.12 (8): p. e0182295.

134. Di Bona, D., et al., Efficacy of allergen immunotherapy in reducing the likelihood of developing new allergen sensitizations: a systematic review. Allergy, 2017. 72 (5): p. 691-704.

135. Zielen, S., et al., Sublingual immunotherapy provides long-term relief in allergic rhinitis and reduces the risk of asthma: A retrospective, real-world database analysis. Allergy, 2018.73 (1): p. 165-177.

136. Jacobsen, L., et al., Specific immunotherapy has long-term preventive effect of seasonal and perennial asthma: 10-year follow-up on the PAT study. Allergy, 2007. 62 (8): p. 943-8.

137. Valovirta, E., et al., Results from the 5-year SQ grass sublingual immunotherapy tablet asthma prevention (GAP) trial in children with grass pollen allergy. J Allergy Clin Immunol, 2018.141 (2): p. 529-538 e13.

138. Prokopakis, E.P., L. Kalogjera, and A.D. Karatzanis,Pediatric Severe Chronic Upper Airway Disease (P-SCUAD). Curr Allergy Asthma Rep, 2015. 15 (12): p. 68.

139. Mathioudakis, A.G., et al., Research Priorities in Pediatric Asthma: Results of a Global Survey of Multiple Stakeholder Groups by the Pediatric Asthma in Real Life (PeARL) Think Tank. J Allergy Clin Immunol Pract, 2020. 8 (6): p. 1953-1960.e9. 